\title{
Gas Phase Sensors for Bases Using Rhodamine B in Nafion Films
}

\author{
Eunhae Hwang, Igor A. Levitsky, William B. Euler \\ Department of Chemistry \\ University of Rhode Island \\ 51 Lower College Road \\ Kingston, RI 02881
}

Correspondence to weuler@chm.uri.edu

Additional Supporting Information may be found in the online version of this article.

Grant sponsor: NSF grant number 0730115 and DHS Center of Excellence Program

Keywords: Nafion, Rhodamine B, sensors, FTIR, UV-vis spectroscopy

\begin{abstract}
A sensor and dosimeter specific to inorganic and organic amine bases composed of Rhodamine B dissolved in Nafion is prepared and evaluated. UV-vis, emission, and IR spectroscopies are used to monitor the presence of a variety of analytes, including aliphatic and aromatic amines. The Rhodamine B serves as a visual indicator of the presence of an analyte by turning from brown to pink upon exposure to aliphatic amines after sufficient exposure, thus acting as a visual monitor. The Rhodamine B also acts as a fluorescent sensor, which turns on upon exposure to the analyte. The sulfonic acid groups in the Nafion protonate the bases, giving unique signatures in the IR spectra allowing for differentiation of the bases.
\end{abstract}

\section{Introduction}

There has been significant work done recently in sensor development for many different types of analytes. ${ }^{1-7}$ Of particular interest to our group has been in finding new materials and sensor platforms for the detection of gas phase molecules. ${ }^{8-11}$ One class of molecules that are of current interest are weak bases such as ammonia, hydrazine, and organic amines. ${ }^{11-13}$ Amines are ubiquitous and are used in chemical and pharmaceutical manufacturing, are products of food decomposition, and are markers for air pollution or disease. Hydrazine is used in fuel for rockets. All of these applications have need for sensitive, selective, and fast sensors that can detect the presence of the base.

In this paper we describe a simple methodology that uses an acid-base reaction as the transducer in a sensor platform. Nafion is used as the acid, but also serves as the mechanical platform for the sensor. Rhodamine B dye is added to the Nafion as a reporting element that can be detected optically when the amine reacts with the sulfonic acid group in the Nafion. The Rhodamine B serves as a turn-on fluorescent detector and changes color, giving a built-in redundancy for the 
sensor and providing a dosimetry function. The structure of the protonated base in the Nafion, as determined by IR spectroscopy, is unique and gives the sensor selectivity, as well. Thus, the Rhodamine B portion of the sensor provides fast response to high doses of the analyte while the Nafion acts as an accumulator, allowing the dosimetry function while also providing analyte differentiation.

Nafion has been highly studied ${ }^{4-25}$ because it has a number of interesting properties that have
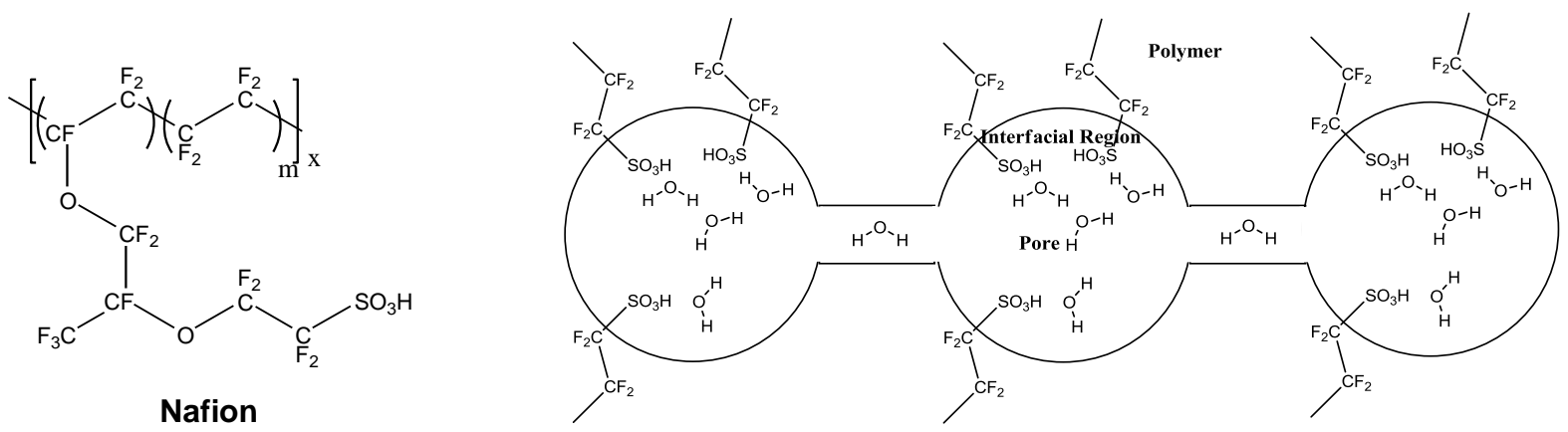

Scheme 1

made it useful in fuel cells and other applications. The chemical structure of Nafion is shown on the top of Scheme 1. The Nafion used in this work has an equivalent weight of 1100 so that $\mathrm{m}$ 6.6 and the concentration of the sulfonic acid groups in a solid film is about $1.3 \mathrm{M}$. The perfluorocarbon backbone of the polymer structure gives Nafion good chemical robustness, which is useful in many applications. Owing the electron withdrawing character of the omnipresent fluorine atoms, the sulfonic acid group is considered a superacid with a $\mathrm{pKa} \sim-6$. $^{26}$ The sulfonic acid group is effective for hydrogen bonding to water so Nafion films are always hydrated, which leads to an interesting pore structure, as depicted in the structure on the bottom in Scheme 1. Although the specific structure is not fully resolved, the water/sulfonic acid groups form pores with sizes of a few nm connected by smaller channels. ${ }^{15,22,24,25}$ This leads to films with three chemically identifiable regions: polymer fluorocarbon, aqueous, and a hydrated sulfonic acid interface. The pore channels have been found to align with the direction of solvent evaporation. $^{24}$ This suggests that Nafion can be used as an acid to develop sensors for bases with a large effective sensory volume if a transducer can be found.

Rhodamine B also has been long studied ${ }^{27-40}$ because it is an effective laser dye and has been used as a sensory material. The dye is typically used in the monocation form $\left(\mathrm{RhBH}^{+}\right)$, shown on the left in Scheme 2, but is protonated to the dication $\left(\mathrm{RhBH}_{2}{ }^{2+}\right)$ at very low $\mathrm{pH}$, shown on the right in Scheme 2. At higher $\mathrm{pH}$ values the carboxylic acid group is deprotonated to give a zwitterion. At concentrations greater than $0.1 \mathrm{mM}$ aggregation occurs that modulates the spectroscopic properties of the dye. Monoprotonated Rhodamine B has a strong fluorescence, with a quantum yield greater than 0.9 in some solvents, ${ }^{30,32}$ and is sensitive to its environment ${ }^{35}$, ${ }^{37-39}$ while the diprotonated form is nonemissive. 
<smiles>CCN(CC)c1ccc2c(-c3ccccc3C(=O)O)c3ccc(=[N+](CC)CC)cc-3oc2c1</smiles>

cation<smiles>CCN([Hg])c1ccc2c(-c3ccccc3C(=O)O)c3ccc(=[N+](CC)CC)cc-3oc2c1</smiles>

protonated dication

Rhodamine B

\section{Scheme 2}

Nafion blended with a sensory dye is an attractive platform for a sensor. The porous structure of the Nafion provides entry point for analytes and the nano-sized hydrophilic pockets can act as accumulation volumes. The channel structure allows facile diffusion into the polymer, and in the case of a basic analyte, easy access for reaction with sulfonic acid sites. If the sensory dye is kept at low concentration, self interaction between the dye molecules can be eliminated. Such a sensor has several possible motifs for detecting an analyte: 1) direct spectroscopic detection of the analyte as it accumulates in the Nafion; 2) detection of the changes in the Nafion structure, especially the water structure as the analyte interacts with the sulfonic acid - in this case the Nafion acts as the transducer; or 3) by detecting changes in the embedded dye that interacts with the analyte directly or by changes in solvation around the dye. We use multiple techniques that probe each of these mechanisms of transduction. For some analytes, the UV spectrum of the analyte can be directly detected as accumulation occurs in the Nafion. For other analytes, there is a change in the water structure in the hydrophilic pockets that can be observed by IR spectroscopy. Finally, for some analytes changes in the visible absorption spectrum or emission spectrum of the Rhodamine $\mathrm{B}$ dye is observed. Owing to the competition between the Nafion sulfonic acid groups and the $\mathrm{RhBH}_{2}{ }^{2+}$, the color change is delayed in the Rhodamine $\mathrm{B}$, proving the dosimeter function.

The analytes studied here are ammonia, aniline, dimethylamine, N,N-dimethylaniline, hydrazine, 2,6-lutidine, phenol, and pyridine. Except for phenol, which is a volatile weak acid used as a control, the analytes are weak bases of varying volatility and base strength. In this paper we report the IR, UV-vis, and emission spectra of the Nafion/Rhodamine B sensor upon exposure to vapors of the analytes listed above.

\section{Experimental}

Materials. Nafion was purchased as a 5\% solution in alcohols, EW 1100, from Sigma-Aldrich and used as received. Rhodamine B chloride was purchased from Baker and recrystallized from methanol. Aniline, dimethylamine (40\% in water), N,N-dimethylaniline, 2,6-lutidine, phenol, 
and pyridine were purchased from Sigma-Aldrich or Fisher Scientific and used as received. Concentrated ammonia solution (Fisher) was diluted to $1 \mathrm{M}$ in water.

Film fabrication. A stock solution of $1.0 \times 10^{-4} \mathrm{M}$ Rhodamine $\mathrm{B}$ in methanol was prepared. Typically, $0.2 \mathrm{~mL}$ of the Rhodamine B solution was mixed with $0.4 \mathrm{~mL}$ of the Nafion solution in $30 \mathrm{~mL}$ beaker and allowed to dry in an oven at $60{ }^{\circ} \mathrm{C}$ for about $120 \mathrm{~min}$ or until the solvent had evaporated. The film was carefully transferred from the beaker to a porous styrofoam substrate for final air drying at room temperature and then placed on an IR card holder (Perkin Elmer).

Measurements. Film thicknesses were estimated using a Motic 350 microscope (Edmunds Scientific) by placing the film edge-on under the objective and by massing a film of known surface area and density $\left(1.40 \mathrm{~g} / \mathrm{mL}^{18}\right)$. The two techniques agreed with $10 \%$ with typical film thicknesses between 5 and $10 \mu \mathrm{m}$. IR spectra were measured on a Perkin-Elmer 1650 FTIR between 4400 and $450 \mathrm{~cm}^{-1}$ at $2 \mathrm{~cm}^{-1}$ resolution. UV-vis spectra were measured on a PerkinElmer Lambda 900 spectrometer between 700 and $200 \mathrm{~nm}$ at $0.5 \mathrm{~nm}$ intervals and a $2 \mathrm{~nm}$ slit width. Emission spectra were measured using an Ocean Optics S2000 spectrometer at $0.4 \mathrm{~nm}$ resolution using an Oriel tungsten halogen light source and monochromator. Excitation was done at $530 \mathrm{~nm}$. All measurements were done at room temperature; the effect of temperature on the sensor performance was not evaluated.

\section{Results and Discussion}

Nafion films were formed by casting from alcohols onto a glass surface. Typical film thicknesses were $5-10 \mu \mathrm{m}$. The concentration of Rhodamine B in the cast films corresponded to $\sim 1000$ sulfonic acid groups per Rhodamine B molecule. Owing to the super acid character of the Nafion, it is expected that nearly all of the Rhodamine B molecules are protonated in the film. The concentration of $\mathrm{RhB}$ is high enough to anticipate aggregation but, as demonstrated by the spectroscopy, this does not occur significantly. Presumably, the suppression of aggregation is due to the pore structure of the Nafion host.

Figure 1 shows the IR and UV-vis spectra of Nafion films with and without Rhodamine B. Wet films containing Rhodamine B are a light pink in color but after drying the films become orange to brown. No emission is found from freshly prepared films. All of these observations are consistent with the Rhodamine B being present in the protonated, dication, monomeric form.

The IR spectrum of Nafion in the $2500-3700 \mathrm{~cm}^{-1}$ region is diagnostic of the water and sulfonic acid structure in the Nafion pores. ${ }^{14,16,17,19-21,23}$ In the absence of any cationic species the spectrum is broad and nearly featureless. For our samples, the maximum is at $3510 \mathrm{~cm}^{-1}$ and the spectrum can be deconvoluted into three broad peaks: 3510,3329 , and $3098 \mathrm{~cm}^{-1}$, all affiliated with $\mathrm{OH}$ stretching vibrations. There also features near $1635 \mathrm{~cm}^{-1}$ assigned to $\mathrm{OH}$ bending modes. Upon addition of Rhodamine B to the film, there are small changes in the $\mathrm{OH}$ vibrations but no other changes in the spectrum (there is a weak peak at $1441 \mathrm{~cm}^{-1}$ that might be attributed to Rhodamine B). In the $\mathrm{OH}$ stretching region several new features become observable and the spectrum deconvolutes into five peaks at 3501, 3243, 3065, 2955, and $2746 \mathrm{~cm}^{-1}$. The major peak near $3500 \mathrm{~cm}^{-1}$ is assigned to absorption of bulk water in the pores and is expected to be sensitive to solvation changes in the Nafion film. The relatively minor changes in the IR 
spectrum caused by the introduction of the Rhodamine $\mathrm{B}$ (band shape changes in the $\mathrm{OH}$ region and a small new peak in the aromatic region at $\sim 1450 \mathrm{~cm}^{-1}$ attributed to the Rhodamine B ring) suggest that the dye molecule is located in the interfacial region (shown in Scheme I) of the Nafion structure. This is consistent with previous findings that polarizable molecules with large size and low charge density, such as Rhodamine B, locate in the interfacial regions. ${ }^{16}$

Electronic spectra of Nafion films are featureless in the visible region but do have a tail in the UV region. Films containing Rhodamine B are orange to brown after drying. The UV-vis spectra shown in the lower portion of Figure 1 confirms the assignment of protonated, dicationic, monomeric Rhodamine B in the films. The three peak structure in the visible region with a maximum at $498 \mathrm{~nm}$ matches the aqueous solution spectrum of Rhodamine $\mathrm{B}$ at $\mathrm{pH}<1 .{ }^{27}$ Deconvolution of the spectrum gives the three components of the spectrum at 467, 498, and 536 $\mathrm{nm}$. These results indicate that there are no electronic perturbations due to quantum confinement in the Nafion pores. The dried Nafion films containing Rhodamine B show no luminescence with any excitation between 400 and $550 \mathrm{~nm}$. The lack of emission is consistent with the Rhodamine $\mathrm{B}$ being present nearly exclusively as the dication in the Nafion films. 

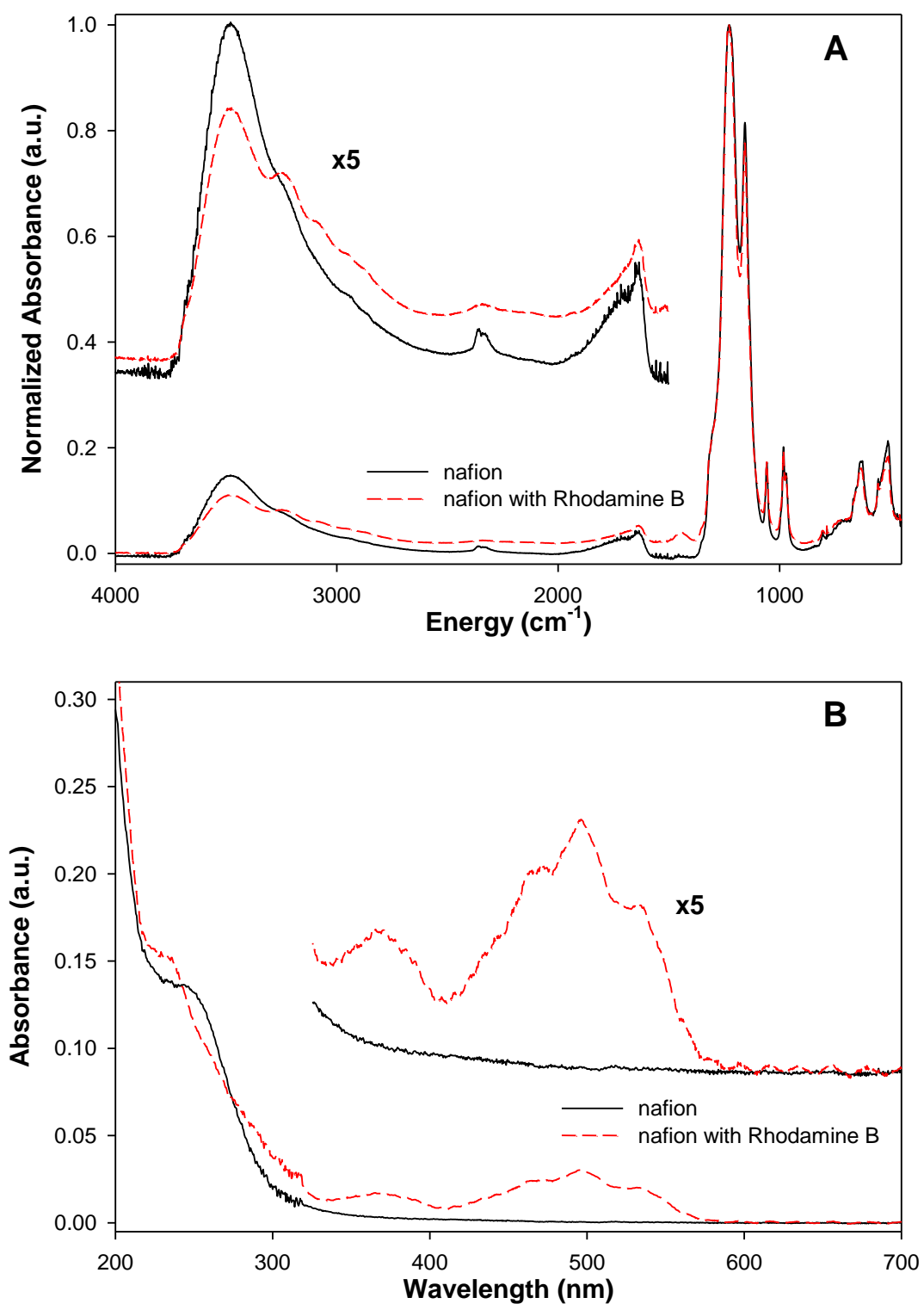

Figure 1. A (top) shows the IR spectra of a Nafion film (black, solid line) and a Nafion film with Rhodamine B added (red, dashed line). The IR spectra are baseline corrected and normalized to the most intense peak at $1228 \mathrm{~cm}^{-1}$. The inset shows a $\times 5$ expansion of the IR region where water absorption peaks are found. Note the better resolution of the features in the $2500-3500 \mathrm{~cm}-1$ region of the film containing Rhodamine $\mathrm{B}$, indicative of the presence of a cationic species in the hydrophilic pockets of the Nafion. B (bottom) shows the UV-vis spectra of a Nafion film (black, solid line) and a Nafion film with Rhodamine $\mathrm{B}$ added (red, dashed line). The inset shows a $\times 5$ expansion of the visible region where the primary Rhodamine B absorption occurs. The three maxima centered at $498 \mathrm{~nm}$ indicate that the Rhodamine B is in the dication form. 
Figure 2 shows the changes in the IR spectra of a film exposed to dimethylamine vapors. In less than $10 \mathrm{sec}$ the spectrum in the $1500-3700 \mathrm{~cm}^{-1}$ changes drastically, demonstrating the large change in the structure of the water in the Nafion pores. The dimethylamine reacts with the acids in the film and forms dimethylammonium ion, which changes the structure of the water in the Nafion pores considerably, as indicated by the three resolved features in the $2800-3500 \mathrm{~cm}^{-1}$ regions (the peak maxima are 3500,3125 , and $2835 \pm 5 \mathrm{~cm}^{-1}$ ), assigned to $\mathrm{OH}$ (and perhaps

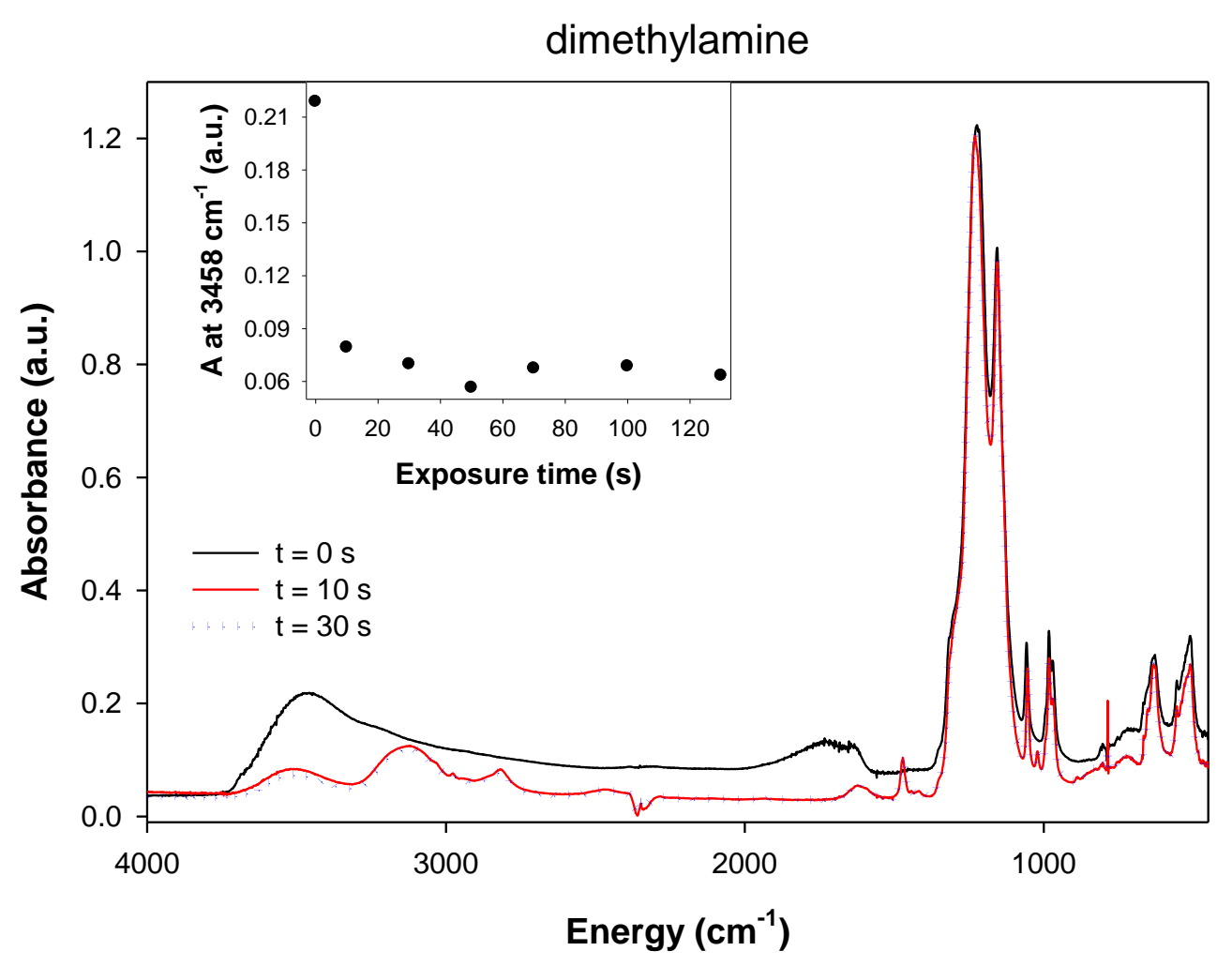

Figure 2. IR spectra of a Nafion/Rhodamine B film exposed to dimethylamine vapor. The inset shows the intensity change of the $\mathrm{OH}$ stretching maximum at $3548 \mathrm{~cm}^{-1}$ as a function of exposure time.

some $\mathrm{CH}$ ) stretching vibrations of solvated dimethylammonium cations) and the sharpening of the bending modes in the $1500-1700 \mathrm{~cm}^{-1}$ region.

The dimethylamine causes large changes in the UV-vis properties of Rhodamine $\mathrm{B}$, as demonstrated in Figure 3. In the visible region a peak at $555 \mathrm{~nm}$ grows (Fig. 3A) while the feature centered at $598 \mathrm{~nm}$ diminishes. This demonstrates that the dimethylamine is deprotonating the dicationic Rhodamine B creating the monocation form. This is confirmed by the emission spectra shown in Fig. 3B. The emission peak at $570 \mathrm{~nm}$, affiliated with the monocation form of Rhodamine $\mathrm{B},{ }^{27}$ grows with exposure to dimethylamine. 

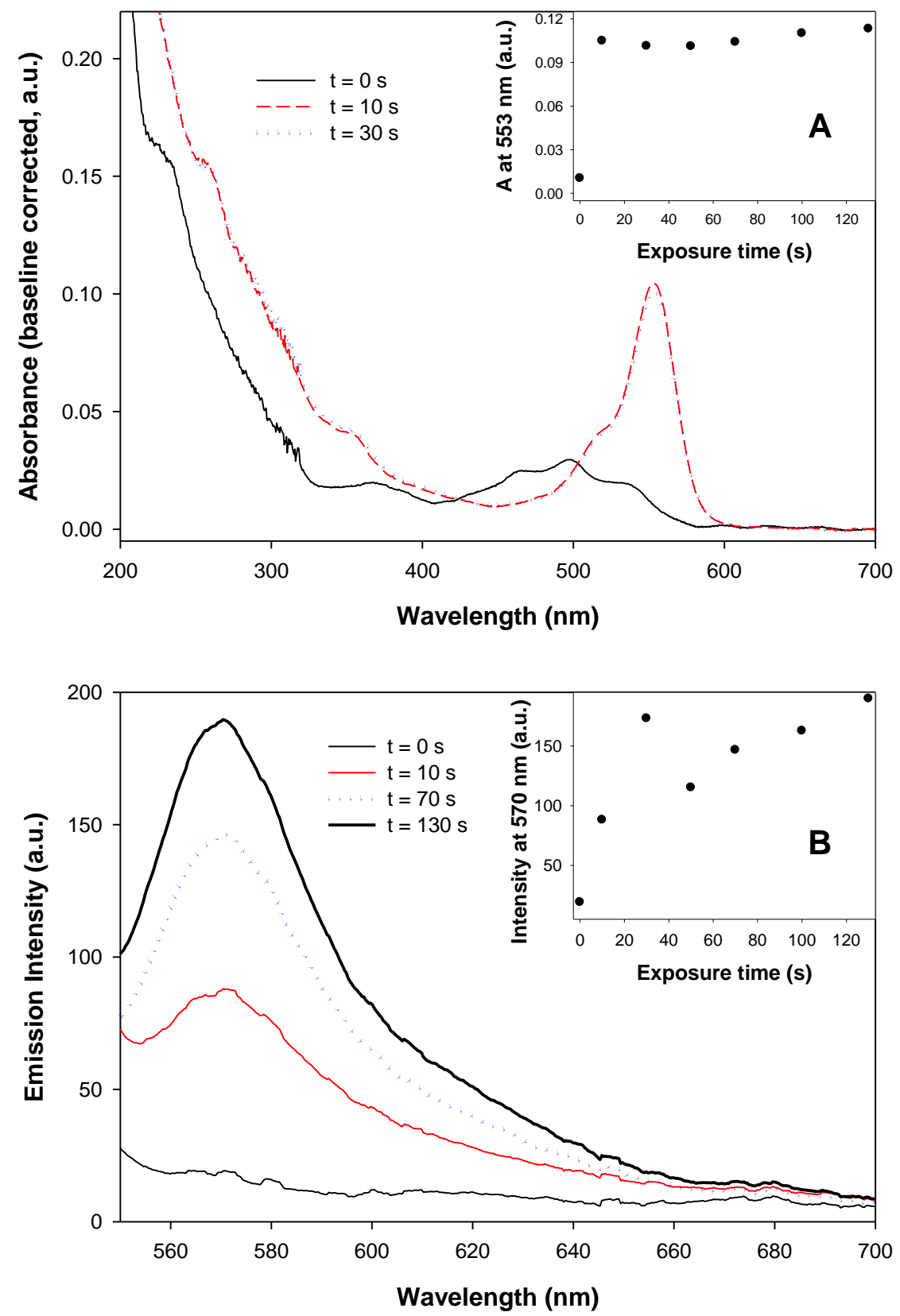

Figure 3. A (top) UV-vis spectra of a Nafion/Rhodamine B film exposed to dimethylamine vapors. The inset shows the intensity change of the peak at $555 \mathrm{~nm}$ as a function of exposure time. B (bottom) Emission spectra of a Nafion/Rhodamine B film exposed to dimethylamine vapors. The inset shows the intensity change of the $570 \mathrm{~nm}$ peak as a function of exposure time.

The spectral changes from both the Nafion and Rhodamine B probes indicate that dimethylamine becomes protonated upon exposure to the composite films. Both the sulfonic acid and the Rhodamine B serve as acid sources. The dimethylammonium cation is solvated in the hydrophilic pores of the Nafion and is likely located in or near the interfacial region of the Nafion. 

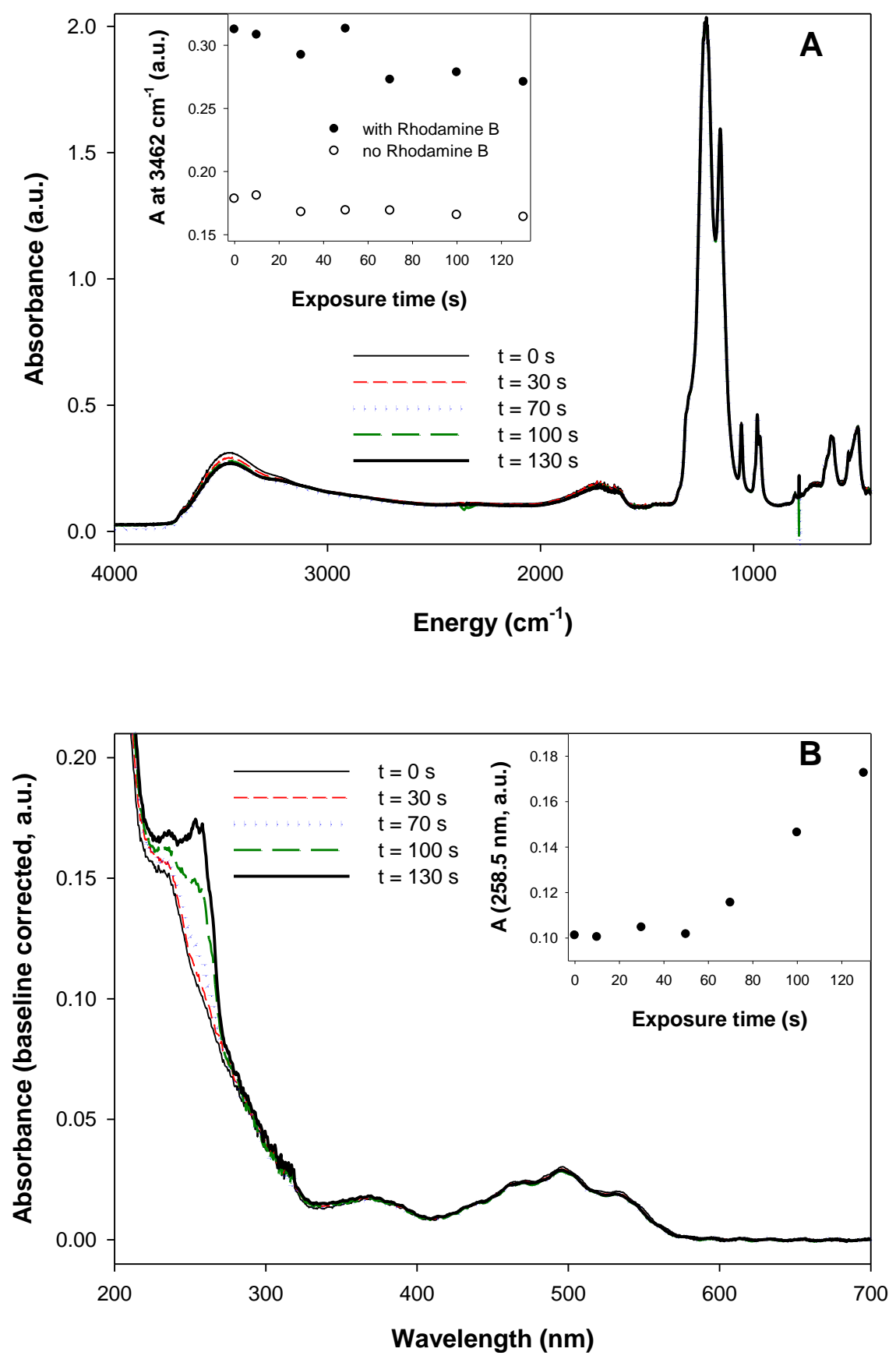

Figure 4. A (top) IR spectra of Nafion/Rhodamine B film as a function of exposure to phenol. The inset shows the intensity change of the OH stretching peak at $3462 \mathrm{~cm}^{-1}$ as a function of exposure time for a film containing Rhodamine B (black circles) and for a film with no Rhodamine B (open circles). B (bottom) UV-vis spectra of Nafion/Rhodamine B film as a function of exposure to phenol. The inset shows the intensity change of the peak at $258.5 \mathrm{~nm}$ as a function of exposure time. 
Exposure of the Nafion/Rhodamine B film to an acid, phenol, gives completely different spectral responses, as shown in Figure 4. The IR spectra (Fig. 4A) show essentially no change when phenol is admitted to the composite film. There is a slight reduction in the intensity of the $\mathrm{OH}$ stretching maximum at $3462 \mathrm{~cm}^{-1}$, which is observed for films either with or without Rhodamine B. This indicates a very modest solvation change in the hydrophilic pockets and/or interfacial region of the Nafion. No acid-base reaction is expected so the only interactions between the phenol and the water in the pores should be via weak hydrogen bonding. This conclusion is supported by the UV-vis spectra shown in Fig. 4B. There is no change in the Rhodamine B features in the visible region and there is no luminescence observed, indicating that the phenol neither reacts with the dye nor even weakly binds to the dye. The UV region of the spectrum does show a band with increasing intensity at $258.5 \mathrm{~nm}$, which is attributed to absorption from the phenol trapped in the pores in the film. There is a short induction time before the phenol band becomes observable on the sloping background, but grows linearly thereafter. Phenol is weakly

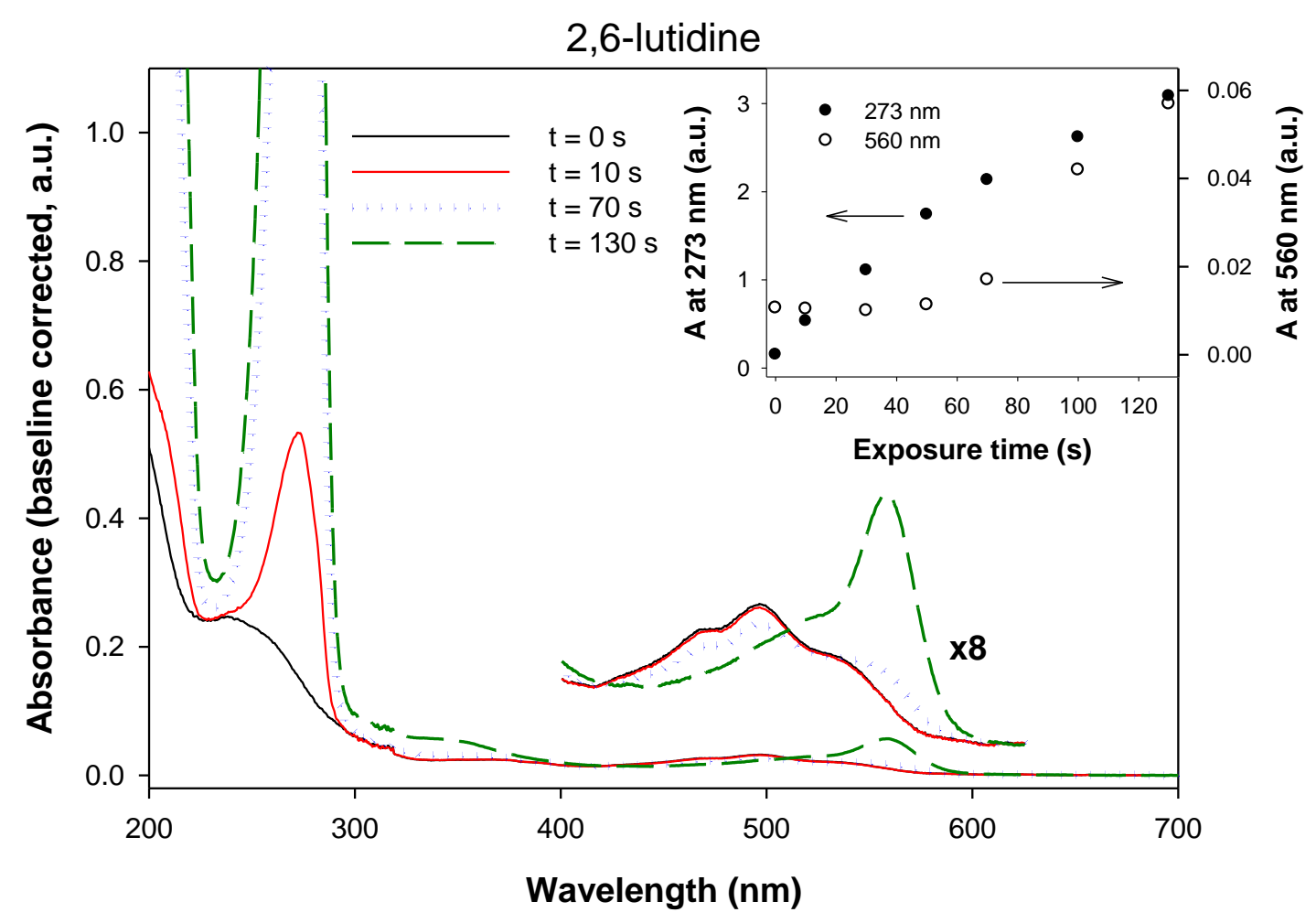

Figure 5. UV-vis spectral changes upon exposure of the Nafion/Rhodamine B sensor to 2,6-lutidine. The inset shows the time dependence of the peaks at $273 \mathrm{~nm}$ (black circles, left axis) and $560 \mathrm{~nm}$ (open circles, right axis).

solvated in the aqueous pockets and otherwise does not strongly interact with the Nafion or Rhodamine B. This is what is expected for a weak acid, a control, where no significant interaction with the sensor components is observed. 
Exposure of the composite films to 2,6-lutidine gives another response pattern. The changes in the IR spectra and emission spectra are similar to that observed for exposure of dimethylamine. In the IR region, there are significant changes in the $1500-3600 \mathrm{~cm}^{-1}$ spectral region, indicative of changes in the water structure in the Nafion. There are no changes in the IR spectra below $1500 \mathrm{~cm}^{-1}$, demonstrating that the fluoropolymer portion of the Nafion does not absorb the analyte (spectra can be found in the Supporting Materials, Figure S8). In the emission spectra, the peak at $570 \mathrm{~nm}$ grows as the Rhodamine B dication deprotonates into the monocation, just as with the dimethylamine (Supporting Materials, Figure S9). However, in the UV-vis spectra the 2,6-lutidine, Figure 5, shows a substantially different response than the dimethylamine. In the UV region a peak at $273 \mathrm{~nm}$, attributed to a $\pi-\pi^{*}$ transition in the aromatic ring of 2,6-lutidine, grows rapidly, easily distinguishable after $10 \mathrm{sec}$ of exposure. After a short lag of about $70 \mathrm{sec}$, a peak at $560 \mathrm{~nm}$ grows while the feature centered at $494 \mathrm{~nm}$ diminishes. The latter changes are associated with the deprotonation of the Rhodamine B dication, confirming the changes observed in the emission spectra. Thus, in the case of the aromatic base the sensor has a dual response in the UV-vis spectrum: direct detection of the analyte because of accumulation in the aqueous pores and indirect detection based on the acid-base reaction with Rhodamine B. The delay in the response of the Rhodamine $\mathrm{B}$ is attributed to the relative acid strength of the protonated Rhodamine B vs. the sulfonic acid groups in the Nafion: the sulfonic acid is a stronger acid so must have all of its protons depleted before the Rhodamine B can react with the basic analyte.

The sensor versatility is demonstrated by the response to all of the analytes tested here. All bases show changes in the emission spectrum with growth of the peak at $570 \mathrm{~nm}$. The responses in the emission spectra are sensitive and relatively fast, ranging from under $10 \mathrm{sec}$ to about $120 \mathrm{sec}$. The UV-vis spectra can distinguish between the aromatic and nonaromatic bases. The aromatic bases accumulate in the aqueous pores and the $\pi-\pi^{*}$ transition of the aromatic ring can be easily observed in the $250-280 \mathrm{~nm}$ region. All of the basic analytes except aniline and N,Ndimethylaniline react with the Rhodamine $\mathrm{B}$ dication to give the monocation, with the accompanying change in the visible region: growth of a peak at $560 \mathrm{~nm}$ and loss of the feature at $494 \mathrm{~nm}$. The aromatic bases tend to promote the change in the visible region slowly, on the order of $100 \mathrm{sec}$, while the nonaromatic bases react rapidly, in less than $10 \mathrm{sec}$. 


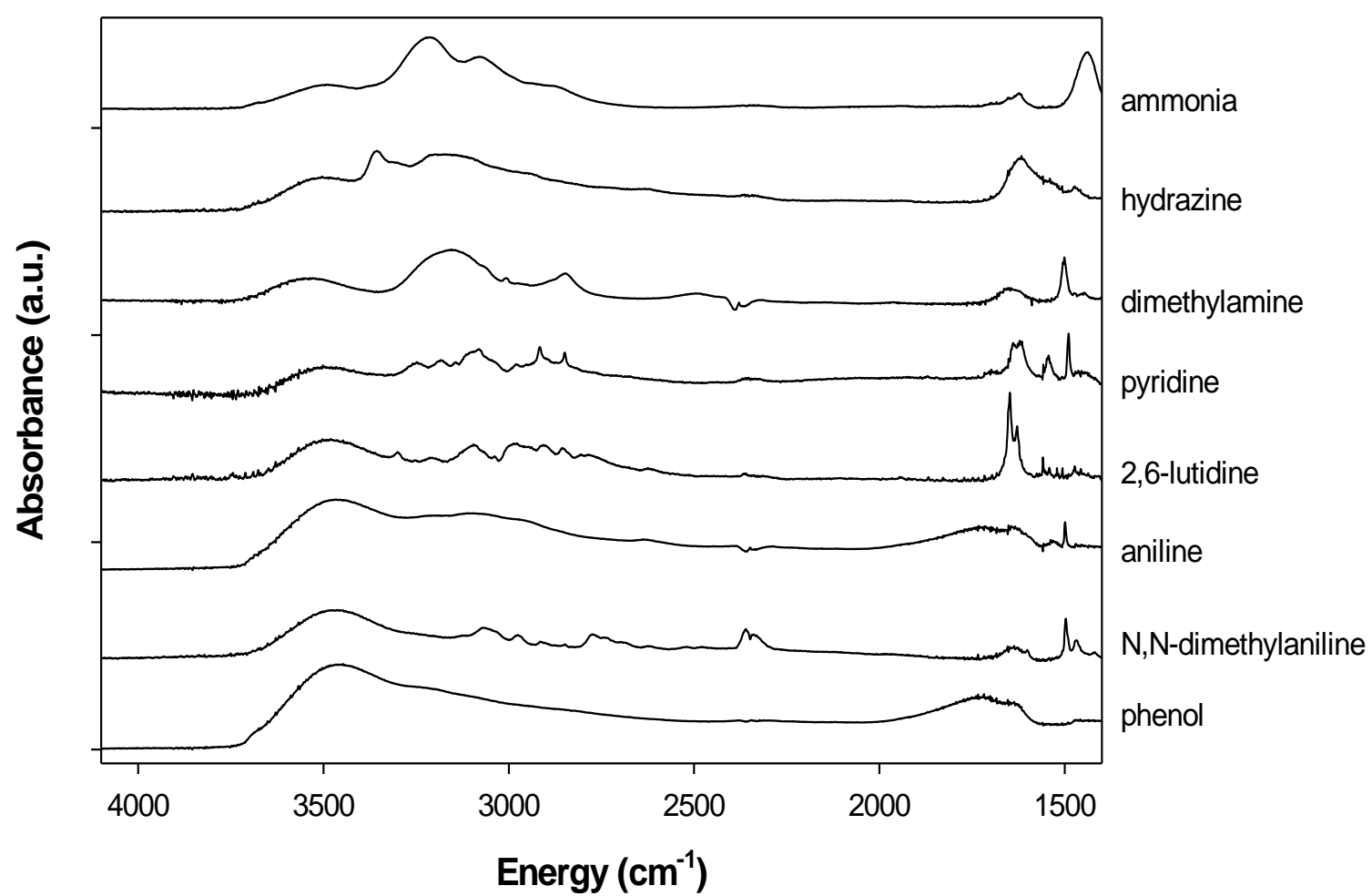

Figure 6. IR spectra of the Nafion/Rhodamine B sensor after exposure to the indicated analyte. Each sensor film was exposed to the analyte until no further spectral changes were observed, from $10 \mathrm{sec}$ to $300 \mathrm{sec}$, depending upon the analyte.

The IR spectra provide much more selectivity, as shown in Figure 6. Each analyte affects the structure of the hydrated pocket in the Nafion, modifying both the $\mathrm{OH}$ stretching and bending modes and adding N-H bands, both stretching and bending. Each analyte has a unique spectrum in the $1400-4000 \mathrm{~cm}^{-1}$ range so that the sensor can differentiate the bases. In contrast, the IR spectrum of phenol, the reference acid, is unmodified with respect to a Nafion film.

The sensitivity of the sensor is based on the exposure time. Since the Nafion pores act to accumulate the analyte, eventually a response can be detected by any amount of analyte. For concentrations less than $1 \mathrm{ppm}$ this takes many hours. However, since the Nafion pores capture the bases irreversibly, the accumulation can continue indefinitely, which allows this sensor system to act as an effective dosimeter. This means that ultimate sensitivity is related to exposure time and that the sensor can detect analytes below the ppm level for long enough exposure times (hours). The binding between the analyte bases and the strong acids used in this sensor is sufficient to prevent reversibility.

Table 1. Properties of the analytes used in this study.

\begin{tabular}{|l|c|c|c|c|}
\hline Analyte & $\begin{array}{c}\text { Water } \\
\text { Solubility }\end{array}$ & $\mathrm{pKa}$ & $\begin{array}{c}\text { Vapor Pressure } \\
\left(25^{\circ} \mathrm{C}, \text { bar }\right)\end{array}$ & $\begin{array}{c}\text { Rate constant } \\
\left(\mathrm{IR}, \mathrm{s}^{-1}\right)\end{array}$ \\
\hline Dimethylamine $(\sim 40 \%)$ & High & 10.77 & $0.16^{41}$ & 0.24 \\
\hline Ammonia (1 M) & High & 9.25 & $0.016^{42}$ & 0.029 \\
\hline Hydrazine & High & 7.92 & $0.019^{43}$ & 0.070 \\
\hline 2,6-Lutidine & $\sim 4 \mathrm{M}$ & 6.65 & $0.0074^{44}$ & 0.040 \\
\hline Pyridine & High & 5.17 & $0.027^{45}$ & 0.23 \\
\hline
\end{tabular}




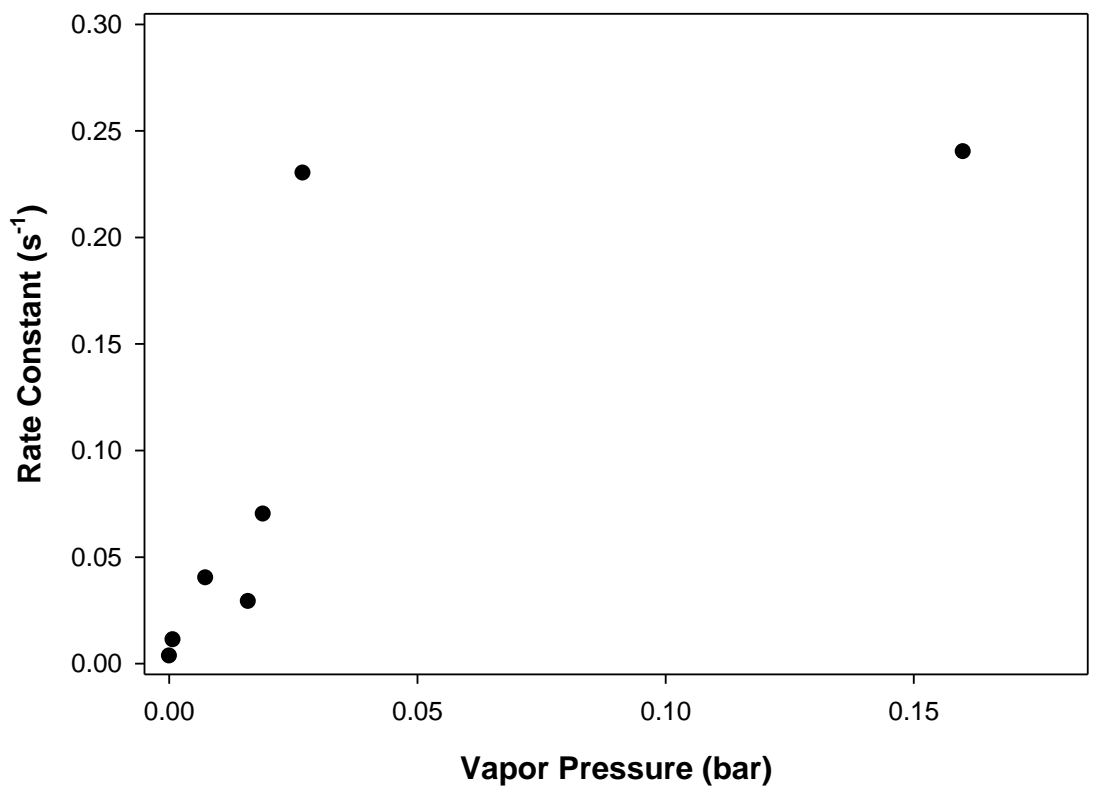

Figure 7. The first order rate constant for each analyte determined by the IR data plotted as a function of analyte room-temperature vapor-pressure.

\begin{tabular}{|l|c|c|c|c|}
\hline N,N-Dimethylaniline & $\sim 0.2 \mathrm{M}$ & 5.15 & $0.00010^{46}$ & 0.0034 \\
\hline Aniline & $\sim 0.4 \mathrm{M}$ & 4.85 & $0.00084^{47}$ & 0.011 \\
\hline
\end{tabular}

The response of the sensor depends on several characteristics of the analyte, including the vapor pressure, the $\mathrm{pKa}$, the solubility in water, and the diffusion in the Nafion pores. The analytes with the highest vapor pressure give the fastest response, as expected, since more base is available to diffuse into the sensor. To test this assumption, the first order rate constant was determined using the IR data for each analyte (given in Table 1). The evaluated rate constants are plotted as a function vapor pressure in Figure 7, which shows a reasonably good correlation between the vapor pressure and the rate constant. The outlier is for pyridine, which also has a high solubility in water suggesting that the solubility of the analyte in the Nafion pores also plays an important role in the sensor response. This observation points to two conclusions. First, the water in the Nafion pores is acting as bulk water, not nanoconfined water. Second, the limiting rate for the sensor response is likely the diffusion of the analyte through the pores to reach the acidic sites. The acid/base reaction, either with sulfonic acid groups or the Rhodamine B is expected to be fast.

The mechanism of the sensor response can be summarized as follows, where A is the analyte:

$$
\begin{aligned}
& \mathrm{A}(g) \stackrel{\mathrm{A}}{ }(\text { pore }) \\
& \mathrm{A}(\text { pore })+\mathrm{RSO}_{3} \mathrm{H} \rightarrow \mathrm{AH}^{+}(\text {pore })+\mathrm{RSO}_{3}^{-} \\
& \mathrm{A}(\text { pore })+\mathrm{RhBH}_{2}{ }^{2+}(\text { interface }) \rightarrow \mathrm{AH}^{+}(\text {pore })+\mathrm{RhBH}^{+}(\text {interface })
\end{aligned}
$$


The IR spectra probe reaction (2) directly and imply that reaction (1) is the rate limiting step, as suggested by the data in Figure 7. The UV-vis and emission spectra monitor reaction (3). The lag time observed in the optical response is caused by the analyte preferentially reacting with the stronger acid, the sulfonic acid on the Nafion. Only after a substantial portion of the sulfonic acid groups in any given pore are consumed does reaction with the diprotonated Rhodamine B commence. In this mechanism, one of the roles of the Nafion is to protonate the Rhodamine B into the dicationic state, which requires a strong polymer acid.

\section{Conclusion}

We describe here a simple sensor platform for detection of gas phase bases. Nafion is used as the mechanical support but the pore structure of the Nafion also provides ready access of the analyte to the acidic species in the sensor. Rhodamine B is introduced into the Nafion thin film to give an optical transducer. Detection by IR, UV-vis, and emission spectroscopies show different responses for different analytes. Aromatic bases accumulate in the Nafion pores and can be directly detected by their $\pi-\pi^{*}$ transition the UV spectral region. After a lag time, changes in the Rhodamine B dye can be monitored in both the emission and visible spectra, which provides a dosimeter function to the material. IR spectra probe the changes in the solvation of the sulfonic acid groups in the interfacial region of the Nafion pores and are unique for each analyte.

\section{References}

1. Dasgupta, P. K.; Genfa, Z.; Li, J.; Boring, C. B.; Jambunathan, S.; Al-Horr, R. Anal. Chem., 1999, 71, 1400.

2. Gromov, S. P.; Ushakov, E. N.; Vedernikov, A. I.; Lobova, N. A.; Alfimov, M. V.; Strelenko, Y. A.; Whitesell, J. K.; Foxe, M. A. Org. Lett., 1999, 1, 1697.

3. Chen, L.; McBranch, D. W.; Wang, H.-L.; Helgeson, R.; Wudl, F.; Whitten, D. G. Proc. National Acad. Sci., 1999, 96, 12287.

4. Jenkins, A. L.; Uy, O. M.; Murray, G. M. Anal. Chem., 1999, 71, 373.

5. McQuade, D. T.; Pullen, A. E.; Swager, T. M. Chem. Rev., 2000, 100, 2537.

6. Zhang, S.-W.; Swager, T. M. J. Am. Chem. Soc., 2003, 125, 3420.

7. Rose, A.; Zhu, Z.; Madigan, C. F.; Swager, T. M.; Bulović, V. Nature, 2005, 434, 876.

8. Levitsky, I. A.; Krivoshkylov, S. G.; Grate, J. W. Anal. Chem., 2001, 73, 3441.

9. Levitsky, I. A.; Krivoshkylov, S. G.; Grate, J. W. J. Phys. Chem. B, 2001, 105, 8468. 
10. Kamarchuk, G. V.; Kolobov, I. G.; Khotkevich, A. V.; Yanson, I. K.; Pospelov, A. P.; Levitsky, I. A.; Euler, W. B. Sens. Actuat. B Chem., 2008, 134, 1022.

11. Gao, T.; Tillman, E. S.; Lewis, N. S. Chem. Mater., 2005, 17, 2904.

12. Preininger, C.; Mohr, G. J. Anal. Chim. Acta, 1997, 342, 207.

13. Che, Y.; Yang, X.; Loser, S.; Zang, L. Nano Lett., 2008, 8, 2219.

14. Falk, M. Can. J. Chem., 1980, 58, 1495.

15. Gierke, T. D.; Munn, G. E.; Wilson, F. C. J. Polym. Sci. Part B: Polym. Phys., 1981, 19, 1687.

16. Yeager, H. L.; Steck, A. J. Electrochem. Soc., 1981, 128, 1880.

17. Quezado, S.; T. Kwak, J. C.; Falk, M. Can. J. Chem., 1984, 62, 958.

18. Zook, L. A.; Leddy, J. Anal. Chem., 1996, 68, 3793.

19. Zinger, B.; Shier, P. Sens. Actuators B, 1999, 56, 206.

20. Iwamoto, R.; Oguro, K.; Sato, M.; Iseki, Y. J. Phys. Chem. B, 2002, 28, 6973.

21. Liang, Z.; Chen, W.; Liu, J.; Wang, S.; Zhou, Z.; Li, W.; Sun, G.; Xin, Q. J. Membrane Sci., 2004, 233, 39.

22. Rubatat, L.; Gebel, G.; Diat, O. Macromolecules, 2004, 37, 7772.

23. Basnayake, R.; Peterson, G. R.; Casadonte, Jr., D. J.; Korzeniewski, C. J. Phys. Chem. B, 2006, 110, 23938.

24. Li, J.; Wilsmeyer, K. G.; Madsen, L. A. Macromolecules, 2008, 41, 4555.

25. Schmidt-Rohr, K.; Chen, Q. Nat. Mater., 2008, 7, 75.

26. Ryder, A. G.; Power, S.; Glynn, T. J. Appl. Spec., 2003, 57, 73.

27. Ramette, R. W.; Sandell, E. B. J. Am. Chem. Soc., 1956, 78, 4872.

28. Ferguson, J. ; Mau, A. W. H. Chem. Phys. Lett., 1972, 17, 543.

29. Chambers, R. W.; Kajiwara, T.; Kearns, D. R. J. Phys. Chem., 1974, 78, 380.

30. Karstens, T.; Kobs, K. J. Phys. Chem., 1980, 84, 1871. 
31. Kubin, R. F. ; Fletcher, A. N. J. Luminescence, 1982, 27, 455.

32. Snare, M. J.; Trelvar, F. E.; Ghiggino, K. P.; Thistlewaite, P. J. J. Photochem. 1982, 18, 335.

33. Casey, K. G.; Quitevis, E. L. J. Phys. Chem., 1988, 92, 6590.

34. Arbeloa, F. L.; Ojeda, P. R.; Arbeloa, I. L. J. Luminescence, 1989, 44, 105.

35. Nakashima, K.; Duhamel, J.; Winnik, M. A. J. Phys. Chem., 1993, 97, 10702.

36. Khan, S. S.; Jin, E. S.; Sojic, N.; Pantano, P. Anal. Chim. Acta, 2000, 404, 213.

37. Mchedlov-Petrossyan, N. O.; Vodolazkaya, N. A.; Doroshenko, A. O. J. Fluorescence, 2003, $13,235$.

38. Moreno-Villoslada, I. ; Jofré, M. ; Miranda, V. ; González, R. ; Sotelo, T. ; Hess, S. ; Rivas, B. L. J. Phys. Chem. B, 2006, 110, 11809.

39. Godfrey Alig, A. R.; Gourdon, D.; Israelachvili, J. J. Phys. Chem. B, 2007, 111, 95.

40. Mchedlov-Petrossyan, N. O.; Vodolazkaya, N. A.; Bezkrovnaya, O. N.; Yakubovskaya, A. G.; Tolmachev, A. V.; Grigorovich, A. V. Spectrochim. Acta A, 2008, 69, 1125.

41. Christie, A. O. ; Crisp, D. J. J. Appl. Chem., 1967, 17, 11.

42. Clegg, S. L.; Brimblecomb, P. J. Phys. Chem., 1989, 93, 7237.

43. Scott, D. W.; Oliver, G. D.; Gross, M. E.; Hubbard, W. N.; Huffman, H. M. J. Am. Chem. Soc., 1949, 71, 2293.

44. Herrington, E. F. G.; Martin, J. F. Trans. Faraday Soc., 1953, 49, 154.

45. McCullough, J. P.; Douslin, D. R.; Messerly, J. F.; Hossenlopp, I. A.; Kincheloe, T. C.; Waddington, G. J. Am. Chem. Soc., 1957, 79, 4289.

46. Stull, D. R. Ind. Eng. Chem., 1947, 39, 517.

47. Hutton, W. E.; Hildenbrand, D. L.; Sinke, G. C.; Stull, D. R. J. Chem. Eng. Data, 1962, 7, 229. 